\title{
Effect of ramelteon on insomnia severity: evaluation of patient characteristics affecting treatment response
}

\author{
Makoto Uchiyama $^{1} \cdot$ Shigeru Sakamoto ${ }^{2} \cdot$ Kouji Miyata $^{2}$
}

Received: 1 March 2019 / Accepted: 6 June 2019 / Published online: 15 June 2019

(c) The Author(s) 2019

\begin{abstract}
We conducted a multicenter, open-label, observational study to evaluate the effectiveness and safety of ramelteon in patients with insomnia, and patient characteristics affecting treatment response in routine clinical practice. Eligible patients were aged $\geq 20$ years, had a diagnosis of insomnia with difficulty falling asleep, and were scheduled to receive ramelteon $8 \mathrm{mg} / \mathrm{day}$ for 12 weeks. The primary objective was to evaluate the relationship between changes in insomnia severity [measured by the Insomnia Severity Index (ISI)] and patient characteristics. Changes in the physical (PCS-8) and mental (MCS-8) components of the Short Form-8 (SF-8), and safety were also investigated. 1527 patients received therapy; $40.5 \%$ were aged $\geq 65$ years and 29.7, 56.9, and $13.4 \%$ had mild, moderate, or severe insomnia, respectively. At week 12, significant improvements in ISI score [mean change (standard deviation) from baseline, -5.0 points $(6.3) ; p<.0001]$, PCS-8 score [ +2.40 points $(8.71)$; $p<.0001$ ], and MCS-8 score [+ 3.68 points $(9.47) ; p<.0001]$ were observed. Factors affecting ISI response or remission (based on adjusted odds ratios) included age $\geq 75$ years, shorter disease duration, no alcohol intake, being employed full time, not taking concomitant medication for insomnia, and taking ramelteon more than $1 \mathrm{~h}$ before bedtime. In total, $5.9 \%$ of patients reported adverse drug reactions. In routine clinical practice, 12 weeks' therapy with ramelteon was effective at improving insomnia severity, with no new safety concerns identified. Several patient characteristics were found to influence response to therapy.
\end{abstract}

Clinical trial identifier: UMIN000013271

Keywords Ramelteon $\cdot$ Insomnia $\cdot$ Clinical study $\cdot$ Patient characteristics $\cdot$ Safety

\section{Introduction}

Insomnia is a common disorder and a major problem in modern society [1]. It leads to fatigue, anxiety, and decreased work performance, and is associated with a significant deterioration in health-related quality of life (HRQL) $[2,3]$. In clinical practice, where drug therapy is warranted, insomnia

Electronic supplementary material The online version of this article (https://doi.org/10.1007/s41105-019-00224-1) contains supplementary material, which is available to authorized users.

Shigeru Sakamoto

shigeru.sakamoto@takeda.com

1 Department of Neuropsychiatry, Nihon University School of Medicine, 30-1 Oyaguchi Kamimachi, Itabashi-ku, Tokyo 173-8610, Japan

2 Japan Medical Office, Takeda Pharmaceutical Company Ltd., 1-1 Nihonbashihoncho 2-chome, Chuo-ku, Tokyo 103-8668, Japan is generally treated with benzodiazepine receptor agonists (benzodiazepine or non-benzodiazepine hypnotics). Benzodiazepine receptor agonists bind to the gamma-aminobutyric acid (GABA) A receptor and exert sedative, anticonvulsant, and anxiolytic effects, which result in a potent hypnotic effect [4]. However, benzodiazepine receptor agonists are associated with adverse drug reactions (ADRs), such as muscle relaxation, rebound insomnia, amnesia, and withdrawal symptoms [5, 6], which may detrimentally affect patient HRQL [7].

Ramelteon is a selective melatonin receptor agonist with high selectivity for MT1/MT2 receptors [8]. Preclinical and clinical studies have shown that ramelteon is associated with sleep promotion and can accelerate circadian rhythm re-entrainment, leading to improvement of both transient and chronic insomnia without sedative or anxiolytic effects [8-11]. Ramelteon has been used in Japan, the USA, and other countries for the treatment of insomnia with difficulty falling asleep [12-14]. 
Key objectives of insomnia treatment are to improve sleep parameters as well as mitigate the deterioration of mental and physical functions during the daytime [15]. While the efficacy of ramelteon in decreasing sleep latency has been established [16], there are currently no data on the effect of ramelteon on insomnia severity, daytime functional activities, and HRQL. In addition, it remains unclear as to which patient types may benefit from ramelteon therapy. Therefore, we conducted "an exploratory observational study to investigate Patient characteristics of Ramelteon responders (PRam)" to evaluate the effectiveness of ramelteon in improving insomnia severity and HRQL, and to investigate patient characteristics which may affect treatment response to ramelteon in routine clinical practice.

\section{Materials and methods}

\section{Study design}

This multicenter, open-label, exploratory, observational study was conducted to evaluate the relationship between the effectiveness of ramelteon in patients with insomnia and their characteristics. The study consisted of a 12-week observation period during which patients received $8 \mathrm{mg} /$ day of ramelteon before going to sleep.

This study was conducted in accordance with the ethical principles described in the Declaration of Helsinki, the Japanese Ethical Guideline for Epidemiologic Research, and all other applicable laws and regulations. Approval of the ethical review committees for all study sites and written consent from all patients were obtained before study commencement. In this study, off-site monitoring was regularly performed to ensure that the study was properly conducted, and a risk-based audit was performed to assure the reliability of results of the study.

\section{Patients}

Patients eligible to be enrolled in this study were aged $\geq 20$ years, had a diagnosis of insomnia with difficulty in falling asleep, and were scheduled to receive ramelteon as routine clinical practice. Patients were required to complete the 7-item, self-reported Insomnia Severity Index (ISI) questionnaire and the 8-item Short Form Health Survey (SF-8) HRQL scale within 14 days before the start of ramelteon administration. Patients were excluded if they had received ramelteon within 4 weeks before the start of the study or if they had dementia, severe hepatic impairment, a history of drug abuse, or unstable psychiatric disease. Concomitant use of fluvoxamine maleate was prohibited; the use of other hypnotic drugs was permitted to ensure that the study reflected the real-world setting. Baseline severity of insomnia was assessed by investigators, taking into consideration patients' complaints, sleep parameters, and ISI scores.

\section{Endpoints and assessments}

The primary endpoint was the relationship between patient characteristics and the effectiveness of ramelteon in decreasing insomnia severity, as assessed by the ISI questionnaire (Japanese version; range 0-28; higher score suggests more severe insomnia; patients with a score of $\geq 10$ are considered to have insomnia) [17, 18]. Secondary endpoints included the effectiveness of ramelteon in improving HRQL, as assessed by the SF-8 questionnaire (Japanese version; higher score suggests better HRQL; a score of 50 is considered normal for the Japanese general population), which comprises a physical component summary (PCS-8) and mental component summary (MCS-8) [19].

Patients with an ISI score change from baseline to week 12 (or end of treatment) between the minimum value and value of the first quartile were defined as responders to ramelteon treatment; other patients were defined as nonresponders. Among patients with a baseline ISI score of $\geq 10$, an ISI score of $<10$ at week 12 (or end of treatment) was defined as being in remission (remitters); other patients were defined as non-remitters for post hoc analysis.

Safety was assessed by evaluating incidence of adverse events (AEs), classified using the Medical Dictionary for Regulatory Activities (MedDRA) version 19.0. AEs judged by investigators to have a causal relationship with ramelteon were defined as ADRs.

\section{Statistical analysis}

Patients who received at least one dose of ramelteon were included in the full analysis set; patients with ISI evaluations at week 12 (or end of study) were included in analyses of the relationships between response and patient characteristics. ISI and SF-8 score comparisons between baseline and week 12 were performed by paired $t$ test. Crude odds ratios and $95 \%$ confidence intervals (CI) were calculated using a univariate logistic regression analysis for responders and remitters using patient characteristics related to insomnia as categorical variables. These characteristics were age, gender, severity of insomnia, duration of insomnia, alcohol intake per week, use of a 'nightcap' (alcoholic beverage before bedtime) instead of hypnotics, daily caffeine ingestion, smoking status, employment status, night-shift worker status, concurrent medical conditions, use of anti-insomnia drugs discontinued within 4 weeks before the study, concomitant use of anti-insomnia drugs, and ramelteon dosing timing. These characteristics were chosen as each is generally accepted as being linked to insomnia. Adjusted odds ratios (AORs) and 95\% CIs were calculated using multiple logistic regression 
analysis for responders and remitters using the same characteristics as covariates.

\section{Results}

\section{Patients}

The study was conducted at 99 study sites (primarily clinics with some general hospitals) in Japan between April 2014 and May 2016. Of the 2210 patients who gave consent to participate in the study, 681 patients were excluded due to the potential lack of data reliability based on the audit results and 2 patients were excluded due to the lack of ramelteon administration. Therefore, 1527 were eligible for analysis. Among these patients, 1050 (68.7\%) patients completed the 12-week observation period. Reasons for discontinuation included voluntary withdrawal [188 (39.2\%)], being lost to follow up [118 (24.6\%)], AEs [75 (15.7\%)], and lack of effectiveness [65 (13.6\%)].

Mean age of patients was 56.8 years and $66.3 \%$ were female (Table 1). Patients had insomnia for a median of 1.2 years; most had moderate disease severity $(56.9 \%)$. Regarding lifestyle factors, $62.9 \%$ of patients did not consume any alcohol, $77.3 \%$ of patients were non-smokers, and $65.7 \%$ of patients had a caffeine consumption of $\geq 2$ cups a day. The most frequent concomitant medical condition was hypertension $(26.9 \%$ ) (Table 1 ). Overall, $14.7 \%$ of patients had previously taken an anti-insomnia drug that was discontinued within 4 weeks before the start of the study and $46.6 \%$ took a concomitant anti-insomnia drug during the study (Table 2).

The mean [standard deviation (SD)] duration of ramelteon therapy (defined as the time between the first and last dose of ramelteon during the study period) was 72.0 (35.3) days; $62.6 \%$ of patients had a treatment duration of between 71 and 99 days (Table 2). Regarding drug adherence to ramelteon therapy, $66.7 \%$ of patients took $\geq 90 \%$ of prescribed doses and $17.0 \%$ took $50-89 \%$ of doses.

\section{Change in Insomnia Severity Index score}

At treatment initiation (i.e. at baseline, $n=1520$ ), mean (SD) ISI score was $15.0(5.2)$; at week $12(n=1175)$, it was 10.0 (6.2). Of the 1171 patients with an ISI assessment both at baseline and week 12, the mean (SD) change from baseline to week 12 [-5.0 (6.3)] was statistically significant (two-sided 95\% CI $-5.4,-4.6 ; p<.0001$ ) (Table 3), representing an improvement in patients' disease severity. The minimum value of change in ISI score was -28.0 points and the change in ISI score in the first quartile was -9.0 points (Table 3 ); thus, patients with an ISI score change of between -28.0 and -9.0 were defined
Table 1 Patient demographics and disease characteristics at baseline

\begin{tabular}{|c|c|}
\hline & $N=1527$ \\
\hline Age, years, mean (SD) & $56.8(19.1)$ \\
\hline$\geq 65$ years, $n(\%)$ & $619(40.5)$ \\
\hline Female, $n(\%)$ & $1013(66.3)$ \\
\hline Duration of insomnia, years, median (range) & $1.2(0.0-60.3)$ \\
\hline \multicolumn{2}{|c|}{ Patients' complaint related to insomnia, $n$ (\%) (duplicated count) } \\
\hline Difficulty falling asleep & $1515(99.2)$ \\
\hline Nocturnal awakening & $893(58.5)$ \\
\hline Early morning awakening & $583(38.2)$ \\
\hline Difficulty staying in deep sleep & $662(43.4)$ \\
\hline \multicolumn{2}{|l|}{ Severity of insomnia, $n(\%)$} \\
\hline Mild & $453(29.7)$ \\
\hline Moderate & $869(56.9)$ \\
\hline Severe & $205(13.4)$ \\
\hline \multicolumn{2}{|l|}{ Alcohol consumption per week, $n(\%)$} \\
\hline 0 & $960(62.9)$ \\
\hline $1-2$ days & $256(16.8)$ \\
\hline $3-5$ days & $140(9.2)$ \\
\hline $6-7$ days & $170(11.1)$ \\
\hline Missing & $1(0.1)$ \\
\hline Nightcap instead of hypnotics, $n(\%)$ & $134(8.8)$ \\
\hline \multicolumn{2}{|l|}{ Daily caffeine ingestion, $n(\%)$} \\
\hline$<2$ cups & $523(34.3)$ \\
\hline$\geq 2$ cups & $1003(65.7)$ \\
\hline Missing & $1(0.1)$ \\
\hline \multicolumn{2}{|l|}{ Smoking status, $n(\%)$} \\
\hline Non-smoker & $1181(77.3)$ \\
\hline Smoker ( $<20$ cigarettes/day) & $256(16.8)$ \\
\hline Smoker ( $\geq 20$ cigarettes/day) & $89(5.8)$ \\
\hline Missing & $1(0.1)$ \\
\hline \multicolumn{2}{|l|}{ Employment status, $n(\%)$} \\
\hline Full-time worker & $373(24.4)$ \\
\hline Part-time worker & $180(11.8)$ \\
\hline Unemployed & $973(63.7)$ \\
\hline Missing & $1(0.1)$ \\
\hline Night-shift worker, $n(\%)$ & $73(4.8)$ \\
\hline \multicolumn{2}{|l|}{ Concurrent medical condition $\mathrm{n}(\%)$} \\
\hline Hypertension & $411(26.9)$ \\
\hline Depression & $297(19.4)$ \\
\hline Hyperlipidemia & $297(19.4)$ \\
\hline Diabetes & $158(10.3)$ \\
\hline Anxiety disorder & $151(9.9)$ \\
\hline Reflux esophagitis & $107(7.0)$ \\
\hline
\end{tabular}

$S D$ standard deviation

as treatment responders according to the protocol of this study (Fig. 1). In total, 307 patients were classified as responders and 864 as non-responders. Among patients whose ISI baseline score was $\geq 10(n=983), 437$ had a reduction in score to $<10$ at week 12 and were classified 
Table 2 Treatment exposure to ramelteon and concomitant medications

\begin{tabular}{lc}
\hline$n(\%)$ & $N=1527$ \\
\hline Duration of exposure to ramelteon, days & \\
$1-70$ & $407(26.7)$ \\
$71-99$ & $956(62.6)$ \\
$\geq 100$ & $136(8.9)$ \\
Missing & $28(1.8)$ \\
Timing of ramelteon doses & \\
At same time everyday & \\
$\geq 1$ h before bedtime & $510(33.4)$ \\
Just before bedtime & $231(15.1)$ \\
According to bedtime & \\
$\geq 1$ h before bedtime & $272(17.8)$ \\
Just before bedtime & $514(33.7)$ \\
Anti-insomnia drugs discontinued before study & \\
Hypnotics & $224(14.7)$ \\
Benzodiazepine hypnotics & $195(12.8)$ \\
Non-benzodiazepine hypnotics & $126(8.3)$ \\
Antidepressants & $86(5.6)$ \\
Antipsychotics & $10(0.7)$ \\
Antiallergic drugs & $10(0.7)$ \\
Other & $5(0.3)$ \\
Concomitant anti-insomnia drugs & $8(0.5)$ \\
Hypnotics & $711(46.6)$ \\
Benzodiazepine hypnotics & $657(43.0)$ \\
Non-benzodiazepine hypnotics & $398(26.1)$ \\
Other & $334(21.9)$ \\
Antidepressants & $35(0.2)$ \\
Antipsychotics & $24(1.6)$ \\
Antiallergic drugs & $6.4)$ \\
Other & \\
\hline
\end{tabular}

${ }^{a}$ Within 4 weeks before the start of the study

as being in remission; 546 patients were, thus, classified as non-remitters.

\section{Health-related quality of life}

Patients had a mean (SD) PCS-8 score of 43.53 (8.59) at baseline, which increased to 45.71 (8.19) at week 12. The change from baseline to week 12 of 2.40 (8.71) was statistically significant (two-sided 95\% CI 1.89, 2.91, $p<.0001$ ), representing an improvement in patients' physical functioning. Similarly, mean (SD) MCS-8 score was 41.99 (9.42) at baseline and 45.81 (8.73) at week 12; the change from baseline to week 12 of 3.68 (9.47) was statistically significant (two-sided $95 \%$ CI 3.13, 4.23, $p<.0001$ ), denoting an improvement in mental HRQL.

\section{Patient characteristics affecting change in Insomnia Severity Index score}

In patients defined as responders, patient characteristics significantly affecting the response to ramelteon treatment, based on AORs, were older age ( $\geq 75$ vs. $<65$ years: AOR 1.98), moderate or severe insomnia severity (vs. mild: AOR 2.37 and 5.40, respectively), shorter disease duration $(<1$ vs. $\geq 5$ years: AOR 0.65), no alcohol intake (vs. alcohol on 3-5 days per week: AOR 0.39), not taking concomitant antiinsomnia medication during the study (vs. taking concomitant medication on study: AOR 0.63), and taking ramelteon more than $1 \mathrm{~h}$ before bedtime (vs. at bedtime: AOR 2.04) (Table 4; Supplementary Fig. S1).

In patients defined as being in remission, patient characteristics significantly affecting the response to ramelteon, based on AORs, were older age (65-74 or $\geq 75$ vs. $<65$ years: AOR 1.72 and 2.95 , respectively), shorter disease duration ( $<1$ vs. $\geq 5$ years: AOR 0.57 ), not having a nightcap instead of hypnotics (vs. yes: AOR 0.52), being a non-smoker (vs. < 20 cigarettes/day: AOR 0.58), being employed full time (vs. being unemployed: AOR 2.02), not taking concomitant anti-insomnia medication during the study (vs. taking concomitant medication on study: AOR 0.75 ), and taking ramelteon more than $1 \mathrm{~h}$ before bedtime (vs. just before bedtime: AOR 1.45) (Table 5; Supplementary Fig. S2).

\section{Safety}

AEs were reported in $10.7 \%$ of patients in the study and ADRs occurred in $5.9 \%$ of patients. The most frequent ADRs were somnolence (1.7\%) and dizziness (1.5\%) (Table 6). AEs that were either mild, moderate, or severe in intensity were reported in $7.5,3.0$, and $0.2 \%$ of patients, respectively. AEs leading to discontinuation were recorded in $4.9 \%$ of patients. Serious AEs occurred in $0.6 \%$ of patients; none were considered to be drug related. There were no deaths during the study.
Table 3 Change in ISI score from baseline to week 12 of treatment with ramelteon

\begin{tabular}{llllllllll}
\hline & $n$ & Mean (SD) & Minimum & Q1 & Median & Q3 & Maximum & 95\% CI & $p$ value* \\
\hline Overall & 1171 & $-5.0(6.3)$ & -28 & -9.0 & -4.0 & -1.0 & 20 & $(-5.4,-4.6)$ & $p<.0001$ \\
\hline
\end{tabular}

$C I$ confidence interval, $I S I$ Insomnia Severity Index, $Q$ quartile, $S D$ standard deviation

*One sided $t$ test with merged responder/non-responders or remitter/non-remitters 
Fig. 1 Patient distribution for change from baseline in ISI score. ISI Insomnia Severity Index

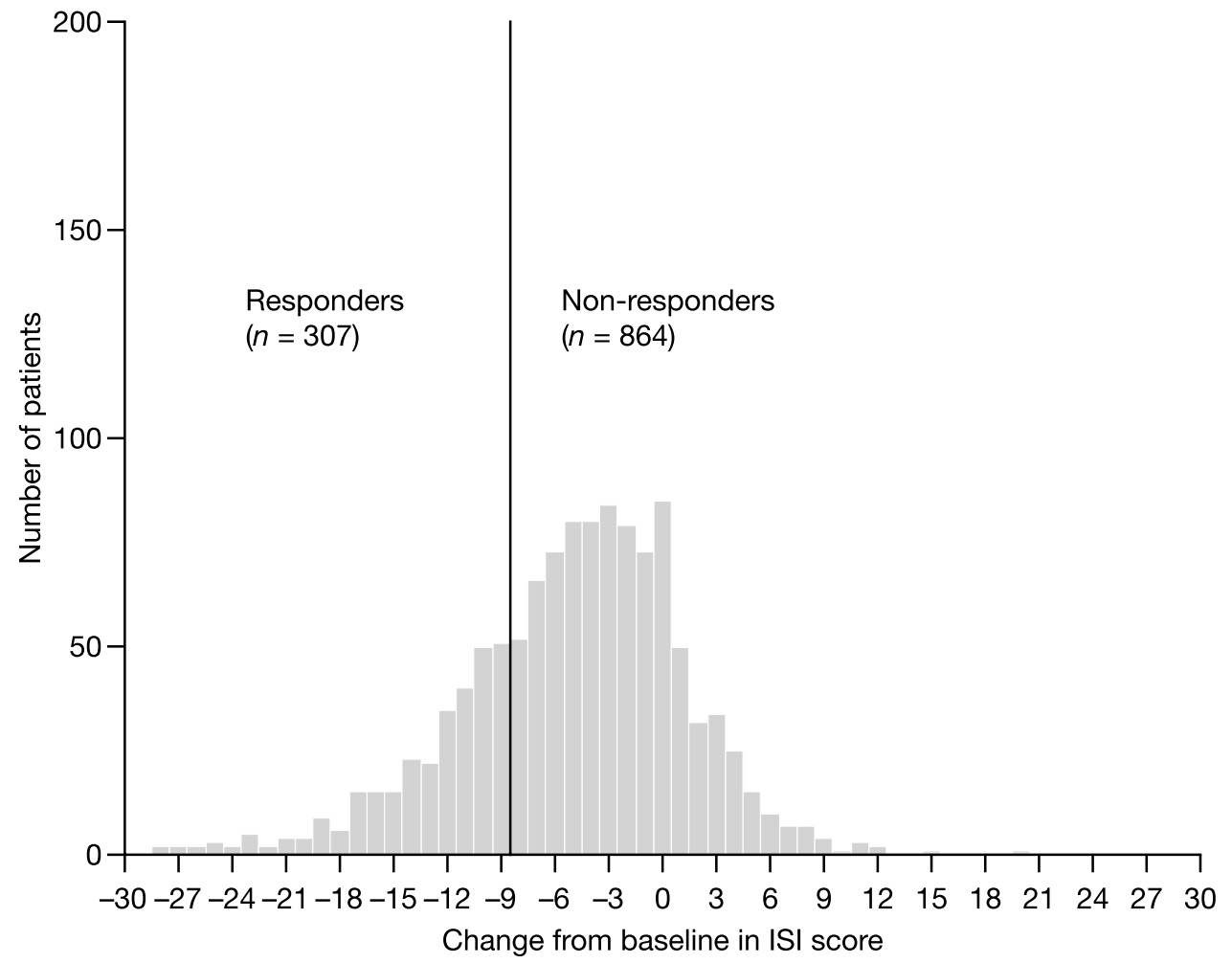

\section{Discussion}

In this observational study, we demonstrated that 12 weeks of ramelteon treatment, as part of routine clinical practice, were associated with statistically significant improvements in both insomnia severity and HRQL, with a tolerable AE profile. In addition, several patient demographic and disease characteristics were found to influence response to ramelteon therapy.

Although the definition of a clinically meaningful change or improvement for ISI score has been discussed, there is no consensus on the definition of a response when using the ISI score [20, 21]. Thus, we defined a responder as a patient whose change in ISI from baseline to week 12 was between the minimum value $(-28.0)$ and value of the first quartile $(-9.0)$, utilizing the methodology for response definition of another clinical study [22]. Consequently, patients with a baseline ISI score of $<9.0$ were defined as non-responders in the 'responder' analysis, even if their scores decreased after ramelteon treatment. To take the response from these patients into account, we performed a post hoc analysis defining patients with an ISI score $\geq 10$ at baseline and $<10$ at week 12 as remitters [23]. While largely congruent, the results of these two analyses did show some differences. For example, multiple logistic regression analysis of responding patients showed that response to ramelteon was more frequent in patients who had moderate or severe insomnia versus those with mild disease. Conversely, multiple logistic regression analysis of remitting patients noted no such association. The rationale behind this discrepancy may lie in the definition of responder employed here, as outlined above. In patients with mild insomnia, the number of patients with a baseline ISI score of $<9.0$ was greater than in those patients who had moderate or severe insomnia (data not shown). Thus, the number of patients who were defined as responders in our analysis was much smaller in the 'mild' group compared with the 'moderate' and 'severe' groups. It, therefore, seems reasonable to interpret these results based on the analysis of remitting patients instead.

Logistic regression analysis in responders showed that the response to ramelteon was significantly higher in older patients aged $\geq 75$ years compared with those aged $<65$ years. In elderly patients, insomnia is thought to be caused by a decrease in melatonin secretion from the pineal gland [24]; thus, it can reasonably be expected that ramelteon will provide better treatment outcomes in this population compared with younger patients, given that it works in a similar way to melatonin [8].

A longer history of insomnia had a negative impact on response rates in both analyses. One possible explanation may be that patient anxiety related to difficulty in falling asleep acts to worsen insomnia in some patients with a long history of insomnia [25]. Because ramelteon has not been shown to have anxiolytic effect [8], one could speculate that earlier use of ramelteon before psychological barriers to sleep develop may be appropriate for achieving better 
Table 4 Relationship between ISI score and patient characteristics in responders versus non-responders

\begin{tabular}{|c|c|c|c|c|c|c|c|}
\hline Factor & Variable & $n$ & Responders; $n(\%)$ & Crude OR & $95 \% \mathrm{CI}$ & Adjusted OR & $95 \% \mathrm{CI}$ \\
\hline \multirow[t]{3}{*}{ Age, years } & $<65$ & 693 & $172(24.8)$ & 1.00 & & 1.00 & \\
\hline & $65-74$ & 203 & $48(23.6)$ & 0.94 & $(0.65,1.35)$ & 1.26 & $(0.80,1.99)$ \\
\hline & $\geq 75$ & 275 & $87(31.6)$ & 1.40 & $(1.03,1.91)^{*}$ & 1.98 & $(1.27,3.09)^{*}$ \\
\hline \multirow[t]{2}{*}{ Gender } & Female & 773 & $196(25.4)$ & 1.00 & & 1.00 & \\
\hline & Male & 398 & $111(27.9)$ & 1.14 & $(0.87,1.50)$ & 1.09 & $(0.80,1.49)$ \\
\hline \multirow[t]{3}{*}{ Severity of insomnia } & Mild & 335 & $53(15.8)$ & 1.00 & & 1.00 & \\
\hline & Moderate & 678 & $188(27.7)$ & 2.04 & $(1.46,2.86)^{*}$ & 2.37 & $(1.66,3.39)^{*}$ \\
\hline & Severe & 158 & $66(41.8)$ & 3.82 & $(2.48,5.88)^{*}$ & 5.40 & $(3.35,8.71)^{*}$ \\
\hline \multirow[t]{4}{*}{ Duration of insomnia, years } & $<1.0$ & 517 & $148(28.6)$ & 1.00 & & 1.00 & \\
\hline & $1.0-2.9$ & 208 & $57(27.4)$ & 0.94 & $(0.66,1.35)$ & 0.90 & $(0.60,1.35)$ \\
\hline & $3.0-4.9$ & 134 & $38(28.4)$ & 0.99 & $(0.65,1.50)$ & 0.91 & $(0.57,1.45)$ \\
\hline & $\geq 5.0$ & 309 & $63(20.4)$ & 0.64 & $(0.46,0.89)^{*}$ & 0.65 & $(0.45,0.96)^{*}$ \\
\hline \multirow[t]{4}{*}{ Alcohol intake per week, days } & 0 & 746 & $207(27.7)$ & 1.00 & & 1.00 & \\
\hline & $1-2$ & 203 & $50(24.6)$ & 0.85 & $(0.60,1.22)$ & 0.84 & $(0.56,1.25)$ \\
\hline & $3-5$ & 100 & $14(14.0)$ & 0.42 & $(0.24,0.76)^{*}$ & 0.39 & $(0.20,0.75)^{*}$ \\
\hline & $6-7$ & 122 & $36(29.5)$ & 1.09 & $(0.72,1.66)$ & 1.02 & $(0.60,1.73)$ \\
\hline \multirow[t]{2}{*}{ Nightcap instead of hypnotics } & No & 1071 & $280(26.1)$ & 1.00 & & 1.00 & \\
\hline & Yes & 100 & $27(27.0)$ & 1.05 & $(0.66,1.66)$ & 1.18 & $(0.66,2.13)$ \\
\hline \multirow[t]{2}{*}{ Daily caffeine ingestion, cups } & $<2$ & 414 & $111(26.8)$ & 1.00 & & 1.00 & \\
\hline & $\geq 2$ & 757 & $196(25.9)$ & 0.95 & $(0.73,1.25)$ & 1.19 & $(0.88,1.60)$ \\
\hline \multirow[t]{3}{*}{ Smoking status, cigarettes/day } & Non-smoker & 913 & $241(26.4)$ & 1.00 & & 1.00 & \\
\hline & $<20$ & 198 & $49(24.7)$ & 0.92 & $(0.64,1.31)$ & 1.01 & $(0.68,1.51)$ \\
\hline & $\geq 20$ & 60 & $17(28.3)$ & 1.10 & $(0.62,1.97)$ & 1.21 & $(0.64,2.29)$ \\
\hline \multirow[t]{3}{*}{ Employment status } & Unemployed & 762 & $204(26.8)$ & 1.00 & & 1.00 & \\
\hline & Part time & 138 & $29(21.0)$ & 0.73 & $(0.47,1.13)$ & 1.05 & $(0.64,1.71)$ \\
\hline & Full time & 271 & $74(27.3)$ & 1.03 & $(0.75,1.40)$ & 1.37 & $(0.92,2.04)$ \\
\hline \multirow[t]{2}{*}{ Night-shift worker } & No & 1124 & $297(26.4)$ & 1.00 & & 1.00 & \\
\hline & Yes & 47 & $10(21.3)$ & 0.75 & $(0.37,1.53)$ & 0.57 & $(0.27,1.24)$ \\
\hline \multirow[t]{2}{*}{ Concurrent depression } & No & 918 & $240(26.1)$ & 1.00 & & 1.00 & \\
\hline & Yes & 253 & $67(26.5)$ & 1.02 & $(0.74,1.40)$ & 1.00 & $(0.69,1.45)$ \\
\hline \multirow[t]{2}{*}{ Concurrent hypertension } & No & 847 & $224(26.4)$ & 1.00 & & 1.00 & \\
\hline & Yes & 324 & $83(25.6)$ & 0.96 & $(0.72,1.28)$ & 0.97 & $(0.66,1.45)$ \\
\hline \multirow[t]{2}{*}{ Concurrent diabetes } & No & 1052 & $285(27.1)$ & 1.00 & & 1.00 & \\
\hline & Yes & 119 & $22(18.5)$ & 0.61 & $(0.38,0.99)^{*}$ & 0.61 & $(0.36,1.06)$ \\
\hline \multirow[t]{2}{*}{ Concurrent hyperlipidemia } & No & 945 & $257(27.2)$ & 1.00 & & 1.00 & \\
\hline & Yes & 226 & $50(22.1)$ & 0.76 & $(0.54,1.07)$ & 0.91 & $(0.59,1.41)$ \\
\hline \multirow[t]{2}{*}{ Concurrent anxiety disorder } & No & 1060 & $284(26.8)$ & 1.00 & & 1.00 & \\
\hline & Yes & 111 & $23(20.7)$ & 0.71 & $(0.44,1.15)$ & 0.79 & $(0.47,1.32)$ \\
\hline \multirow[t]{2}{*}{ Concurrent reflux esophagitis } & No & 1091 & $290(26.6)$ & 1.00 & & 1.00 & \\
\hline & Yes & 80 & $17(21.3)$ & 0.75 & $(0.43,1.29)$ & 0.96 & $(0.52,1.76)$ \\
\hline \multirow{2}{*}{$\begin{array}{l}\text { Anti-insomnia drugs within } 4 \text { weeks } \\
\text { of study start }^{\mathrm{a}}\end{array}$} & No & 992 & $267(26.9)$ & 1.00 & & 1.00 & \\
\hline & Yes & 179 & $40(22.3)$ & 0.78 & $(0.54,1.14)$ & 1.06 & $(0.70,1.60)$ \\
\hline \multirow[t]{2}{*}{ Concomitant anti-insomnia drugs } & No & 598 & $180(30.1)$ & 1.00 & & 1.00 & \\
\hline & Yes & 573 & $127(22.2)$ & 0.66 & $(0.51,0.86)^{*}$ & 0.63 & $(0.47,0.85)^{*}$ \\
\hline \multirow[t]{2}{*}{ Dosing instruction $1^{\mathrm{b}}$} & According to bedtime & 588 & $153(26.0)$ & 1.00 & & 1.00 & \\
\hline & At same time each day & 583 & $154(26.4)$ & 1.02 & $(0.79,1.32)$ & 0.89 & $(0.65,1.20)$ \\
\hline \multirow[t]{2}{*}{ Dosing instruction $2^{\mathrm{b}}$} & Just before bedtime & 566 & $113(20.0)$ & 1.00 & & 1.00 & \\
\hline & $\geq 1 \mathrm{~h}$ before bedtime & 605 & $194(32.1)$ & 1.89 & $(1.45,2.47)^{*}$ & 2.04 & $(1.49,2.78)^{*}$ \\
\hline
\end{tabular}

$C I$ confidence interval, ISI Insomnia Severity Index, $O R$ odds ratio

$* p<.05$

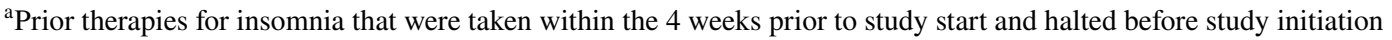

${ }^{\mathrm{b}}$ Dosing instruction 1: dose taken at the same time each day; dosing instruction 2: dose taken more than $1 \mathrm{~h}$ before bedtime 
Table 5 Relationship between ISI score and patient characteristics in remitters versus non-remitters

\begin{tabular}{|c|c|c|c|c|c|c|c|}
\hline Factor & Variable & $n$ & Remitters; $n(\%)$ & Crude OR & $95 \% \mathrm{CI}$ & Adjusted OR & $95 \% \mathrm{CI}$ \\
\hline \multirow[t]{3}{*}{ Age, years } & $<65$ & 589 & $219(37.2)$ & 1.00 & & 1.00 & \\
\hline & $65-74$ & 169 & $80(47.3)$ & 1.52 & $(1.08,2.14)^{*}$ & 1.72 & $(1.12,2.65)^{*}$ \\
\hline & $\geq 75$ & 225 & $138(61.3)$ & 2.68 & $(1.95,3.68)^{*}$ & 2.95 & $(1.90,4.59)^{*}$ \\
\hline \multirow[t]{2}{*}{ Gender } & Female & 650 & $281(43.2)$ & 1.00 & & 1.00 & \\
\hline & Male & 333 & $156(46.8)$ & 1.16 & $(0.89,1.51)$ & 1.13 & $(0.84,1.54)$ \\
\hline \multirow[t]{3}{*}{ Severity of insomnia } & Mild & 222 & $104(46.8)$ & 1.00 & & 1.00 & \\
\hline & Moderate & 607 & $277(45.6)$ & 0.95 & $(0.70,1.30)$ & 1.08 & $(0.77,1.51)$ \\
\hline & Severe & 154 & $56(36.4)$ & 0.65 & $(0.43,0.99)^{*}$ & 0.92 & $(0.58,1.46)$ \\
\hline \multirow[t]{4}{*}{ Duration of insomnia, years } & $<1.0$ & 431 & $217(50.3)$ & 1.00 & & 1.00 & \\
\hline & $1.0-2.9$ & 177 & $79(44.6)$ & 0.79 & $(0.56,1.13)$ & 0.80 & $(0.54,1.18)$ \\
\hline & $3.0-4.9$ & 109 & $46(42.2)$ & 0.72 & $(0.47,1.10)$ & 0.64 & $(0.40,1.02)$ \\
\hline & $\geq 5.0$ & 263 & $93(35.4)$ & 0.54 & $(0.39,0.74)^{*}$ & 0.57 & $(0.40,0.82)^{*}$ \\
\hline \multirow[t]{4}{*}{ Alcohol intake per week, days } & 0 & 626 & $288(46.0)$ & 1.00 & & 1.00 & \\
\hline & $1-2$ & 166 & $71(42.8)$ & 0.88 & $(0.62,1.24)$ & 1.03 & $(0.70,1.52)$ \\
\hline & $3-5$ & 88 & $26(29.5)$ & 0.49 & $(0.30,0.80)^{*}$ & 0.60 & $(0.35,1.03)$ \\
\hline & $6-7$ & 103 & $52(50.5)$ & 1.20 & $(0.79,1.82)$ & 1.43 & $(0.85,2.40)$ \\
\hline \multirow[t]{2}{*}{ Nightcap instead of hypnotics } & No & 892 & $407(45.6)$ & 1.00 & & 1.00 & \\
\hline & Yes & 91 & $30(33.0)$ & 0.59 & $(0.37,0.93)^{*}$ & 0.52 & $(0.29,0.91)^{*}$ \\
\hline \multirow[t]{2}{*}{ Daily caffeine ingestion, cups } & $<2$ & 351 & $166(47.3)$ & 1.00 & & 1.00 & \\
\hline & $\geq 2$ & 632 & $271(42.9)$ & 0.84 & $(0.64,1.09)$ & 1.04 & $(0.78,1.39)$ \\
\hline \multirow[t]{3}{*}{ Smoking status, cigarettes/day } & Non-smoker & 749 & $367(49.0)$ & 1.00 & & 1.00 & \\
\hline & $<20$ & 177 & $54(30.5)$ & 0.46 & $(0.32,0.65)^{*}$ & 0.58 & $(0.39,0.86)^{*}$ \\
\hline & $\geq 20$ & 57 & $16(28.1)$ & 0.41 & $(0.22,0.74)^{*}$ & 0.74 & $(0.39,1.41)$ \\
\hline \multirow[t]{3}{*}{ Employment status } & Unemployed & 651 & $286(43.9)$ & 1.00 & & 1.00 & \\
\hline & Part time & 108 & $41(38.0)$ & 0.78 & $(0.51,1.19)$ & 1.29 & $(0.81,2.07)$ \\
\hline & Full time & 224 & $110(49.1)$ & 1.23 & $(0.91,1.67)$ & 2.02 & $(1.38,2.96)^{*}$ \\
\hline \multirow[t]{2}{*}{ Night-shift worker } & No & 942 & $420(44.6)$ & 1.00 & & 1.00 & \\
\hline & Yes & 41 & $17(41.5)$ & 0.88 & $(0.47,1.66)$ & 0.91 & $(0.45,1.84)$ \\
\hline \multirow[t]{2}{*}{ Concurrent depression } & No & 750 & $357(47.6)$ & 1.00 & & 1.00 & \\
\hline & Yes & 233 & $80(34.3)$ & 0.58 & $(0.42,0.78)^{*}$ & 0.80 & $(0.55,1.15)$ \\
\hline \multirow[t]{2}{*}{ Concurrent hypertension } & No & 719 & $297(41.3)$ & 1.00 & & 1.00 & \\
\hline & Yes & 264 & $140(53.0)$ & 1.60 & $(1.21,2.13)^{*}$ & 1.14 & $(0.77,1.68)$ \\
\hline \multirow[t]{2}{*}{ Concurrent diabetes } & No & 892 & $398(44.6)$ & 1.00 & & 1.00 & \\
\hline & Yes & 91 & $39(42.9)$ & 0.93 & $(0.60,1.44)$ & 0.63 & $(0.38,1.04)$ \\
\hline \multirow[t]{2}{*}{ Concurrent hyperlipidemia } & No & 803 & $348(43.3)$ & 1.00 & & 1.00 & \\
\hline & Yes & 180 & $89(49.4)$ & 1.28 & $(0.93,1.77)$ & 0.99 & $(0.65,1.51)$ \\
\hline \multirow[t]{2}{*}{ Concurrent anxiety disorder } & No & 884 & $401(45.4)$ & 1.00 & & 1.00 & \\
\hline & Yes & 99 & $36(36.4)$ & 0.69 & $(0.45,1.06)$ & 0.92 & $(0.57,1.48)$ \\
\hline \multirow[t]{2}{*}{ Concurrent reflux esophagitis } & No & 918 & $405(44.1)$ & 1.00 & & 1.00 & \\
\hline & Yes & 65 & $32(49.2)$ & 1.23 & $(0.74,2.03)$ & 1.21 & $(0.68,2.14)$ \\
\hline \multirow{2}{*}{$\begin{array}{l}\text { Anti-insomnia drugs within } 4 \text { weeks of } \\
\text { study start }^{\mathrm{a}}\end{array}$} & No & 835 & $376(45.0)$ & 1.00 & & 1.00 & \\
\hline & Yes & 148 & $61(41.2)$ & 0.86 & $(0.60,1.22)$ & 1.00 & $(0.67,1.48)$ \\
\hline \multirow[t]{2}{*}{ Concomitant anti-insomnia drugs } & No & 498 & $251(50.4)$ & 1.00 & & & \\
\hline & Yes & 485 & $186(38.4)$ & 0.61 & $(0.47,0.79)^{*}$ & 0.75 & $(0.56,1.00)^{*}$ \\
\hline \multirow[t]{2}{*}{ Dosing instruction $1^{\mathrm{b}}$} & According to bedtime & 495 & $215(43.4)$ & 1.00 & & 1.00 & \\
\hline & At same time each day & 488 & $222(45.5)$ & 1.09 & $(0.85,1.40)$ & 0.99 & $(0.74,1.32)$ \\
\hline \multirow[t]{2}{*}{ Dosing instruction $2^{\mathrm{b}}$} & Just before bedtime & 461 & $186(40.3)$ & 1.00 & & 1.00 & \\
\hline & $\geq 1 \mathrm{~h}$ before bedtime & 522 & $251(48.1)$ & 1.37 & $(1.06,1.76)^{*}$ & 1.45 & $(1.08,1.94)^{*}$ \\
\hline
\end{tabular}

CI confidence interval, ISI Insomnia Severity Index, OR odds ratio

$* p<.05$

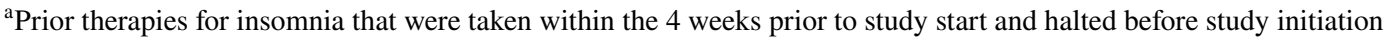

${ }^{\mathrm{b}}$ Dosing instruction 1: dose taken at the same time each day; dosing instruction 2: dose taken more than $1 \mathrm{~h}$ before bedtime 
Table 6 Adverse drug reactions with incidence $\geq 0.2 \%$

\begin{tabular}{lr}
\hline Adverse event, $n(\%)$ & $N=1527$ \\
\hline Somnolence & $26(1.7)$ \\
Dizziness & $23(1.5)$ \\
Malaise & $6(0.4)$ \\
Nausea & $5(0.3)$ \\
Nightmare & $3(0.2)$ \\
Feeling abnormal & $3(0.2)$ \\
\hline
\end{tabular}

treatment outcomes. Further work would be required to explore this concept further.

Employment status, specifically being in full-time employment, was positively associated with response to therapy among patients in remission, with the AOR being significantly higher for full-time workers compared with unemployed patients. A non-significant trend for a positive response to treatment was also observed in the responder analysis. As having a consistent daily routine is linked with improved sleep patterns, it is possible that the environment surrounding a full-time worker makes it easy for them to adjust to the rhythm of, and establish consistent patterns within, their daily life.

In terms of lifestyle factors, alcohol consumption and smoking are well-known causes of insomnia [15]. In the responder analysis, having a weekly intake of no alcohol was associated with response to therapy compared with alcohol intake on 3-5 days per week. A similar, but non-significant trend was observed in the remitter analysis. In patients in remission, having no nightcap instead of hypnotics and nonsmoking status were also both significantly associated with response to ramelteon.

In patients who did receive concomitant anti-insomnia therapies during the study, AORs were significantly lower in both responders and remitters. It should be noted, however, that the category of patients who received concomitant antiinsomnia therapy could have included both those patients for whom a new anti-insomnia drug was added (e.g., due to insufficient response to ramelteon during the 12-week observation period) and patients who started ramelteon as an addition to prior anti-insomnia therapy. Since we did not collect information on the start or end date of concomitant drugs, our analysis was limited, because we could not differentiate between these two statuses. Hence, we are unable to draw any solid conclusions from these data.

Another factor significantly associated with response in both analyses was administration of ramelteon at least $1 \mathrm{~h}$ before bedtime. An aspect that may have contributed to this effect is a decrease in core body temperature $1 \mathrm{~h}$ postadministration of ramelteon, observed in a previous study [26]. The present analysis, thus, suggests that administration of ramelteon at least $1 \mathrm{~h}$ before bedtime is beneficial in terms of improving ISI score. The recommendation for benzodiazepines and non-benzodiazepines is to take these drugs just before bedtime due to safety concerns including amnesia, altered state of consciousness, somnambulism, and hypotonia, which may lead to falls $[5,6]$. Because information on the timing of the onset of AEs was not collected in the present study, we could not assess whether dosing timing of ramelteon was associated with any safety concerns. Further studies are required to more fully assess the impact of dosing timing on the effectiveness and safety of ramelteon therapy.

In terms of safety, our results were similar to those obtained in both clinical investigations [16] and in postmarketing surveillance for ramelteon [27]. No new safety concerns were identified following 12 weeks of treatment in this setting of routine clinical practice.

One key limitation of this study is potential selection bias. This study started 4 years after the launch of ramelteon, so it is likely that many of the investigators would have had experience of ramelteon treatment. Based on the investigators' past clinical experience, patients whom were expected to be responsive to ramelteon and to give consent to participate in the study may have been preferentially selected. These selection biases are thought to have some impact on the result of this study. In addition, the $30 \%$ of exclusion rate from the analysis might influence potential selection bias. The influence of the informed consent process on selection bias could not be determined, as consent rates were not assessed in this study. Finally, as this was a multicenter observational study, it is possible that the investigators' recruitment strategies could have varied.

Another limitation is the absence of a control arm, making it difficult to estimate the degree of improvement compared with untreated patients or those with potential placebo effects. In addition, because most patients had a relatively short duration of disease ( $<1$ year), and thus a shorter experience with other prior or concomitant anti-insomnia therapies, it was difficult to estimate the potential impact of these therapies on patient outcomes.

In this observational study, 12 weeks of therapy with ramelteon $8 \mathrm{mg} /$ day resulted in statistically significant improvements in ISI, PCS-8, and MCS-8 scores from baseline. Several patient characteristics were found to be associated with response or remission following ramelteon treatment, including age $\geq 75$ years, shorter disease duration, no alcohol intake, being employed full time, receiving no concomitant anti-insomnia therapies, and taking ramelteon more than $1 \mathrm{~h}$ before bedtime. In addition, this study identified no new safety concerns when taking ramelteon $8 \mathrm{mg}$ once a day for 12 weeks as part of routine clinical practice.

Acknowledgements The authors would like to thank all patients and their families, and all investigators for their valuable involvement in this study. They would also like to thank Hiroshi Ito of Jikei University 
School of Medicine, Yuichi Inoue of Tokyo Medical University, Naohisa Uchimura of Kurume University School of Medicine, and Tetsuo Shimizu of Akita University Graduate School of Medicine for providing medical advice during development of the protocol. They also acknowledge the contributions of Takashi Okamoto of A2 Healthcare Corporation for his input into the statistical analysis, and Takuma Endo of Takeda Pharmaceutical Company Limited for his input into the study concept and design. Medical writing assistance was provided by Catherine Crookes of FireKite and Takuma Hayashi of Ashfield Healthcare Communications K.K., both Ashfield companies, part of UDG Healthcare plc, during the development of this manuscript, which was funded by Takeda Pharmaceutical Company Limited and complied with Good Publication Practice 3 ethical guidelines (Battisti WP, et al. Ann Intern Med 2015;163:461-4).

Funding This research was supported by Takeda Pharmaceutical Company Limited. The sponsor inputted to the study design, analysis, and interpretation of the data, and reviewed the manuscript for accuracy. The authors had final responsibility for the decision to submit for publication.

\section{Compliance with ethical standards}

Conflict of interest MU has participated in advisory panels and speaker's bureaus for Takeda Pharmaceutical Company Limited. SS and KM are employees of Takeda Pharmaceutical Company Limited.

Ethical committee permission Approval of the ethical review committees for all study sites was obtained before study start.

Research involving human participants and/or animals This study was conducted in accordance with the ethical principles described in the Declaration of Helsinki, the Japanese Ethical Guideline for Epidemiologic Research, and all other applicable laws and regulations.

Informed consent Written informed consent was obtained from all participants before study commencement.

Open Access This article is distributed under the terms of the Creative Commons Attribution 4.0 International License (http://creativeco mmons.org/licenses/by/4.0/), which permits unrestricted use, distribution, and reproduction in any medium, provided you give appropriate credit to the original author(s) and the source, provide a link to the Creative Commons license, and indicate if changes were made.

\section{References}

1. Kim K, Uchiyama M, Okawa M, Liu X, Ogihara R. An epidemiological study of insomnia among the Japanese general population. Sleep. 2000;23(1):41-7.

2. Léger D, Morin CM, Uchiyama M, Hakimi Z, Cure S, Walsh JK. Chronic insomnia, quality-of-life, and utility scores: comparison with good sleepers in a cross-sectional international survey. Sleep Med. 2012;13(1):43-51.

3. Katz DA, McHorney CA. The relationship between insomnia and health-related quality of life in patients with chronic illness. J Fam Pract. 2002;51(3):229-35.

4. Olson RW, DeLorey TM. GABA receptor physiology and pharmacology. In: Siegel GJ, Agranoff BW, Albers RW, Fisher SK, Uhler MD, editors. Basic neurochemistry: molecular, cellular and medical aspects. 6th ed. Philadelphia: Lippincott-Raven; 1999.

5. Agravat A. ' $Z$ '-hypnotics versus benzodiazepines for the treatment of insomnia. Prog Neurol Psychiatry. 2018;22(2):26-9.

6. Dell'osso B, Lader M. Do benzodiazepines still deserve a major role in the treatment of psychiatric disorders? A critical reappraisal. Eur Psychiatry. 2013;28(1):7-20.

7. Combs D, Goodwin JL, Quan SF, Morgan WJ, Shetty S, Parthasarathy S. Insomnia, health-related quality of life and health outcomes in children: a seven year longitudinal cohort. Sci Rep. 2016;6:27921.

8. Miyamoto M. Pharmacology of ramelteon, a selective MT1/ MT2 receptor agonist: a novel therapeutic drug for sleep disorders. CNS Neurosci Ther. 2009;15(1):32-51.

9. Liu J, Clough SJ, Hutchinson AJ, Adamah-Biassi EB, PopovskaGorevski M, Dubocovich ML. MT1 and MT2 melatonin receptors: a therapeutic perspective. Annu Rev Pharmacol Toxicol. 2016;56:361-83.

10. Erman M, Seiden D, Zammit G, Sainati S, Zhang J. An efficacy, safety, and dose-response study of ramelteon in patients with chronic primary insomnia. Sleep Med. 2006;7(1):17-24.

11. Zammit G, Erman M, Wang-Weigand S, Sainati S, Zhang J, Roth T. Evaluation of the efficacy and safety of ramelteon in subjects with chronic insomnia. J Clin Sleep Med. 2007;3(5):495-504.

12. Takeda Pharmaceutical Company Limited. ROZEREM (ramelteon) Prescribing information (Japan). 2010. http://www.pmda. go.jp/files/000153012.pdf. Accessed 15 Apr 2019.

13. Neubauer DN. A review of ramelteon in the treatment of sleep disorders. Neuropsychiatr Dis Treat. 2008;4(1):69-79.

14. Takeda Pharmaceuticals America Inc. ROZEREM (ramelteon) Prescribing information. 2010. http://www.fda.gov. Accessed 15 Apr 2019.

15. Sateia MJ, Buysse DJ, Krystal AD, Neubauer DN, Heald JL. Clinical practice guideline for the pharmacologic treatment of chronic insomnia in adults: an American Academy of Sleep Medicine clinical practice guideline. J Clin Sleep Med. 2017;13(2):307-49.

16. Uchiyama M, Hamamura M, Kuwano T, Nishiyama $H$, Nagata $\mathrm{H}$, Uchimura N. Evaluation of subjective efficacy and safety of ramelteon in Japanese subjects with chronic insomnia. Sleep Med. 2011;12(2):119-26.

17. Bastien $\mathrm{CH}$, Vallieres A, Morin CM. Validation of the Insomnia Severity Index as an outcome measure for insomnia research. Sleep Med. 2001;2(4):297-307.

18. Munesawa T, Morin CM, Inoue Y, Nedate K. Development of a Japanese version of the insomnia severity score questionnaire. Jpn J Psychiatr Treat. 2009;24:219-25.

19. Fukuhara S, Suzukamo Y. Manual of the SF-8 Japanese version. Kyoto: Institute for Health Outcomes and Process Evaluation Research; 2004.

20. Morin CM, Belleville G, Belanger L, Ivers H. The Insomnia Severity Index: psychometric indicators to detect insomnia cases and evaluate treatment response. Sleep. 2011;34(5):601-8.

21. Yang M, Morin CM, Schaefer K, Wallenstein GV. Interpreting score differences in the Insomnia Severity Index: using healthrelated outcomes to define the minimally important difference. Curr Med Res Opin. 2009;25(10):2487-94.

22. Tsukahara K, Kimura K, Morita S, Ebina T, Kosuge M, Hibi K, Okuda J, Iwahashi N, Maejima N, Nakachi T, Ohtsuka F, Hashiba K, Tahara Y, Sugano T, Umemura S. Impact of highresponsiveness to dual antiplatelet therapy on bleeding complications in patients receiving drug-eluting stents. Circ J. 2010;74(4):679-85.

23. Pillai V, Roth T, Roehrs T, Moss K, Peterson EL, Drake CL. Effectiveness of benzodiazepine receptor agonists in the 
treatment of insomnia: an examination of response and remission rates. Sleep. 2017;40(2):zsw044.

24. Olde Rikkert MG, Rigaud AS. Melatonin in elderly patients with insomnia. A systematic review. Z Gerontol Geriatr. 2001;34(6):491-7.

25. Staner L. Sleep and anxiety disorders. Dialogues Clin Neurosci. 2003;5(3):249-58.

26. Markwald RR, Lee-Chiong TL, Burke TM, Snider JA, Wright KP Jr. Effects of the melatonin MT-1/MT-2 agonist ramelteon on daytime body temperature and sleep. Sleep. 2010;33(6):825-31.
27. Uchiyama M, Sakamoto S, Shirai K. Study for safety and efficacy of ramelteon for insomnia patients with difficulty falling sleep-results from post-marketing surveillance study with over 3000 patients. Geriatr Med. 2014;52:813-37.

Publisher's Note Springer Nature remains neutral with regard to jurisdictional claims in published maps and institutional affiliations. 